\title{
Marmatherapie
}

\section{Heilkunst - entwickelt aus der Kampfkunst}

\author{
In MMW Nr. 42/2006, S. 28 berichte- \\ ten wir über eine Studie zur ayurve- \\ dischen Marmamassage bei Schlag- \\ anfallpatienten. Dazu erreichte uns \\ folgender Leserbrief:
}

- Mit der im westlichen Kulturkreis unbekannten Marmatherapie wurden Schlaganfallpatienten behandelt. Als „objektive Variable“ wurde der BarthelIndex zugrunde gelegt. Warum, das bleibt Geheimnis der Autoren. Als „,harter" Endpunkt oder Variable in dieser Studie wären auch die Vigilanz oder die Libido geeignet.

Professor Ernst zeigte aber in seinem Kommentar nicht die groben wissenschaftlichen Fehler in der Publikation auf. Er kommt vielmehr zu dem Fazit, dass "nicht alles, was aus der überlieferten indischen Medizin stammt, so gut ist, wie manche Gurus uns glauben machen". Die Autoren der Studie stammen übrigens wie Ernst von der Peninsula Medical School und zitieren ihn auch im Literaturverzeichnis.

Die Marmatherapie ist Bestandteil der traditionellen Ayurvedamedizin im südindischen Bundesstaat Kerala. Dort wird eine jahrtausendealte ayurvedische Kampf- und Heilkunst in Kalaris gepflegt.

Kalaris sind traditionelle, südindische Ausbildungs- und Behandlungsstätten, in denen Kampf- (Payat) und Heilkunst (Chikitsa) unterrichtet und praktiziert werden. Die Heilkunst, das Kalari Chikitsa, ist auf das uralte Wissen der südindischen Kalarikämpfer zurückzuführen und beeinflusste später maßgeblich die Entwicklung der Ayurvedamassage. Ziel dieser Behandlungsform ist es, die körperlichen und geistigen Funktionen sowie den Energiefluss des Menschen zu optimieren bzw. wiederherzustellen. Dazu dienen sowohl systemische als auch lokale Behandlungstechniken. Diese beinhalten spezielle, aufeinander abgestimmte Massa- ge- und Mobilisationstechniken, therapeutische Übungen sowie thermo- und phytotherapeutische Anwendungen.

Die Kenntnis über Marmas und Nadis wurde während zahlreicher kriegerischer Auseinandersetzungen gewonnen. Man beobachtete, dass der Mensch im Kampf durch Verletzung bestimmter Stellen des Körpers entweder kampfunfähig oder sogar getötet wurde. Aus diesen Kenntnissen entwickelte sich eine gefürchtete Kampfkunst, aber auch Behandlungsmöglichkeiten und eine effektive Kriegs-

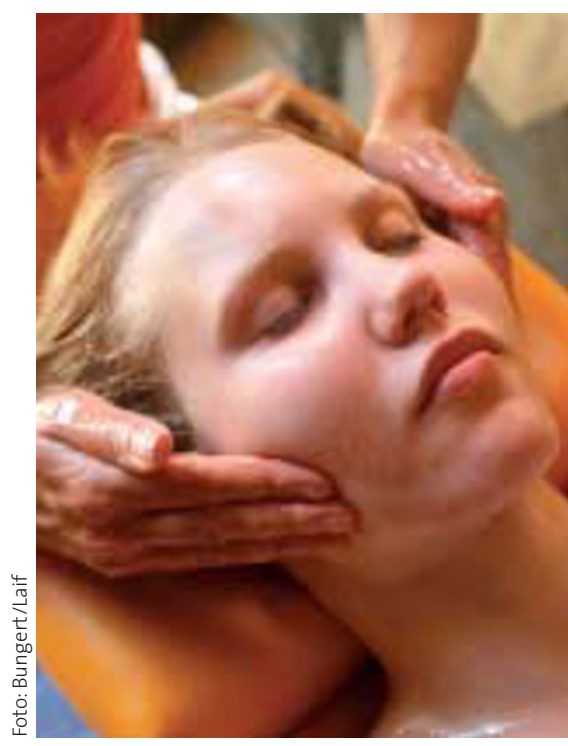

Marmamassage, angeboten in einem Wellnesshotel.

chirurgie, die auch eine Triage nach modernen notfallmedizinischen Kriterien ermöglichte. Wurde ein bestimmter Marma durch einen Pfeil verletzt, konnten die Marmaärzte die Prognose abgeben, dass sich eine Behandlung lohnt oder aber dass der Krieger in nächster Zeit versterben wird. Bei der chirurgischen Behandlung von Kriegsverletzungen wurde vor Schnitten in Marmas ausdrücklich gewarnt. Diese jahrhundertealte, effektive Qualitätssicherung der Marmatherapie bedarf keiner Kontrolle durch westliche Uni- versitäten, sie spricht für sich, denn sie war im wahrsten Sinne überlebenswichtig.

Während der Kolonialbesatzung Indiens durch die Briten waren die Ayurvedamedizin und die Kalari-MarmaTherapie verboten. Behandelten Marmaärzte trotz Verbots ihre Patienten, wurden ihnen die Hände abgehackt.

Ist es Ironie der Geschichte, dass vor den kolonialen Gräueltaten der Briten sich heute eine britische Universität anmaßt, die Wirkungsweise der Marmatherapie zu erforschen?

- Dr. med. Dipl.-Biochemiker Hans-Ulrich Jabs, Facharzt für Innere Medizin, Von-derReck-Straße 3, D-48301 Nottuln

\section{Antwort von Prof. E. Ernst:}

Ich bin Herrn Dr. Jabs sehr dankbar, uns diesen interessanten Hintergrund zur Marmamassage anzubieten. Da die Artikel in der Rubrik „Kritisch Gelesen“ sehr kurz sein sollen, musste ich mich auf das Wesentliche beschränken. Dr. Jabs irrt sich, wenn er meint, die Studie stammt aus meinem „eigenen Haus“. Im Gegenteil: Ich hatte es abgelehnt, mich an diesem Projekt zu beteiligen, sodass es schließlich ohne die Mithilfe meines Lehrstuhls durchgeführt wurde. Ich bin ferner nicht der Meinung, dass aufgrund der historischen Zusammenhänge eine britische Universität sich nicht „anmaßen“ sollte, dieses Thema zu bearbeiten. Im vorliegenden Fall war es sogar (wenn ich richtig informiert bin) Prinz Charles, der auf dieses Projekt erpicht war. Die Tatsache, dass in Dachau einst Häftlinge bei Versuchen zur Kälteexposition umkamen, bedeutet auch nicht, dass dieses Thema für deutsche Forscher tabu ist!

- Prof. E. Ernst, MD, PhD, FRCP, FRCPEd, Complementary Medicine, Peninsula Medical School, Universities of Exeter \& Plymouth, 25 Victoria Park Road, Exeter 